EPJ Web of Conferences 28, 09002 (2012)

DOI: $10.1051 /$ epjconf/20122809002

(C) Owned by the authors, published by EDP Sciences, 2012

\title{
SUSY + Beyond Standard Model Higgs Searches at the Tevatron
}

\author{
Patwa, Abid ${ }^{1, *, a}$ \\ Brookhaven National Laboratory, Upton, New York, USA \\ *On behalf of the CDF and D $\varnothing$ Collaborations
}

\begin{abstract}
Recent results by the CDF and D $\varnothing$ Collaborations for non-Standard Model Higgs boson searches in $p \bar{p}$ collisions at center-of-mass energy of $\sqrt{s}=1.96 \mathrm{TeV}$ using up to $8.2 \mathrm{fb}^{-1}$ of Fermilab Tevatron data are discussed. Searches for neutral Higgs bosons predicted in the Minimal Supersymmetric Standard Model (MSSM), doubly-charged Higgs bosons predicted in extended models, as well as Higgs bosons within Hidden Valley and Fermiophobic models are described.
\end{abstract}

\section{Introduction}

The Standard Model (SM) of particle physics has been very successful in describing particles and their interactions. However, the SM is incomplete and several extensions to the model predict additional Higgs bosons. These extended models lead to a sector where the Higgs behaves similar to the SM Higgs but tends to exhibit different couplings with other particles, and thereby can significantly enhance the branching ratios (BR) of the various Higgs decays. This paper focuses on searches for Higgs bosons by the CDF and D $\varnothing$ Collaborations in $p \bar{p}$ collisions at the Fermilab Tevatron in Run II within four different frameworks: 1) the Minimal Supersymmetric Standard Model (MSSM), 2) extended models which predict doubly-charged Higgs bosons, and Higgs bosons within 3) Hidden Valley and 4) Fermiophobic models.

\section{MSSM Higgs Bosons}

The Higgs sector in MSSM requires two Higgs doublets, which leads to five physical Higgs bosons after electroweak symmetry breaking: two neutral CP-even Higgs $\left(h^{\circ}, H^{\circ}\right)$, one CP-odd Higgs $\left(A^{\circ}\right)$, and a pair of charged Higgs $\left(H^{ \pm}\right)$. The three neutral Higgs are commonly denoted as $\phi$. At tree-level, the MSSM Higgs is fully specified by two free parameters, which are chosen to be the mass of the $\mathrm{CP}$-odd Higgs, $M_{A}$, and the ratio of the vacuum expectation values of the two Higgs doublets, $\tan \beta$. Radiative corrections subsequently introduce additional dependencies on SUSY parameters. The inclusive Higgs boson production cross section $(\sigma)$ is enhanced in the region of low $M_{A}$ by a factor depending on $\tan \beta$. In most regions of the parameter space, $\phi$ decays to $b \bar{b}(\tau \tau)$ pairs with branching ratios on the order of $90 \%(10 \%)$. Although the $\tau$-mode has a smaller $\mathrm{BR}$, it yields a much cleaner signature whereas the $b$-mode tends to suffer from large multijet backgrounds. Nonetheless, both $\mathrm{D} \varnothing$ and CDF study both final states allowing the Tevatron searches to probe several MSSM benchmark scenarios, and thereby extend the region covered by LEP [1].

\footnotetext{
a e-mail: abid@fnal.gov
}

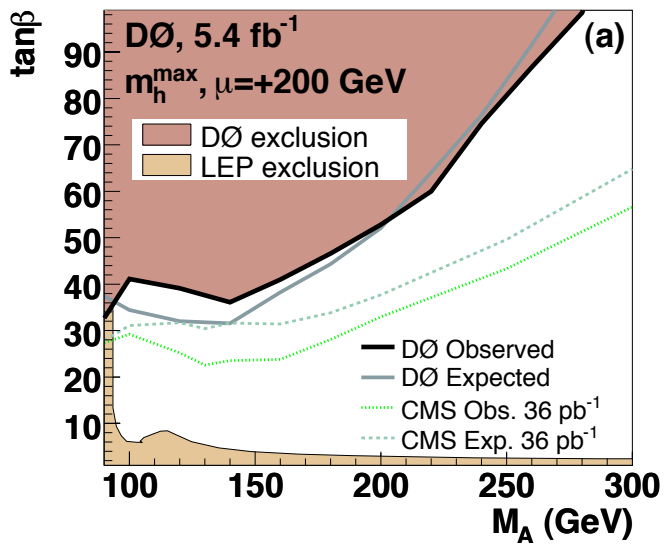

Fig. 1. Exclusions in the $\left(M_{A}, \tan \beta\right)$ plane for the $m_{h}^{\max }$ and $\mu>0$ representative MSSM scenario from the $\phi \rightarrow \tau \tau$ search by DØ.

\section{$2.1 \phi \rightarrow \tau \tau$ Searches}

Searches for $\phi \rightarrow \tau \tau$ have been performed by CDF [2] using $1.8 \mathrm{fb}^{-1}$ of data and require the $\tau$-lepton to decay into $\tau_{e} \tau_{\text {had }}, \tau_{\mu} \tau_{\text {had }}$, and $\tau_{e} \tau_{\mu}$ states, where $\tau_{e}, \tau_{\mu}\left(\tau_{\text {had }}\right)$ are the leptonic (hadronic) decays of the tau. Events with an isolated $e$ or $\mu$ oppositely charged from a $\tau_{\text {had }}$ are selected. Hadronic taus are separated according to their decay mode and selected using a variable-size cone algorithm. Additional selections are imposed to help reject backgrounds including a requirement on the relative direction of the visible $\tau$ decay products and the $\mathbb{E}_{T}$, which suppresses contributions from $W+$ jets. After selections, the irreducible background from $Z \rightarrow \tau \tau$ remains, and the visible mass, defined as the invariant mass of the visible $\tau$ decay products and missing momentum four-vector approximated by $\not P_{T}=\left(\mathbb{E}_{T}, \mathbb{E}_{x}, \mathbb{E}_{y}, 0\right)$ is exploited to search for a $\tau \tau$ resonance above the expected background. Since no significant excess is observed in data, mass-dependent upper limits on $\sigma \times \mathrm{BR}$ are calculated at $95 \%$ C.L. and translated to $\left(M_{A}\right.$, $\tan \beta$ ) exclusions for representative MSSM scenarios.

Considering $5.4 \mathrm{fb}^{-1}$ of data, a recent search by D $\varnothing$ reconstructs $\tau_{\mu} \tau_{\text {had }}$ and $\tau_{e} \tau_{\mu}$ final states [3]. Events with 
isolated and oppositely charged lepton pairs are selected. Hadronic taus are categorized by their decay types and discriminated from the jet background using a neural network (NN) $\tau$-identification algorithm. Data-driven methods using control regions based on the $\mathrm{NN}$ and the charge of the decaying $\tau$-pairs are used to estimate the multijet background contribution. A series of additional selections are imposed to help reduce backgrounds further. Since the data agrees with the expected background across the visible mass spectrum, 95\% C.L. upper limits on $\sigma \times \mathrm{BR}$ are calculated up to $M_{\phi}$ of $300 \mathrm{GeV}$. These limits are interpreted in benchmark MSSM scenarios and reach sensitivities of $\tan \beta \sim 30$ at low $M_{A} \sim 140 \mathrm{GeV}$. The results, shown for the $m_{h}^{\max }$ and $\mu>0$ case in Fig. 1, are comparable to exclusions from searches by the ATLAS [4] and CMS [5] Collaborations each based on $36 \mathrm{pb}^{-1}$ of data.

\section{$2.2 \phi b \rightarrow b \bar{b} b$ Searches}

Given the large multijet background component, a direct $\phi \rightarrow b \bar{b}$ search is difficult. Instead, both CDF and DØ search for $\phi$ production in association with at least one $b$-jet. Using integrated luminosities of $2.6 \mathrm{fb}^{-1}$, CDF selects events with $3 b$-tagged jets [6]. Contributions from heavy-flavor multijet backgrounds and any possible signal are modeled through a two-dimensional fit of the data to the dijet mass spectrum of the two leading jets, $m_{12}$, and a quantity sensitive to the flavor of the jets, $x_{\text {tags }}$. Subsequently, CDF searches for enhancements in $m_{12}$, and massdependent limits are determined for $\sigma \times \mathrm{BR}$, as shown in Fig. 2 (top-left).

Similarly, the $\mathrm{D} \varnothing$ search is based on $5.2 \mathrm{fb}^{-1}$ of data by requiring triple $b$-tagged jets via a NN-based $b$-tagger [7]. The light- and heavy-flavor background composition is estimated from a global fit of MC simulated events to data over 0-, 1-, 2-, and 3-b-tagging points. A six-variable likelihood is used to discriminate Higgs boson $b$-jets from multijet backgrounds. The search sensitivity is improved by separating the analysis into three- and four-jet channels. After all selections, the dijet invariant mass of the two leading jets is used as the input to calculating the $\sigma \times \mathrm{BR}$ limit, which is shown in Fig. 2 (bottom-left).

The limits on $\sigma \times \mathrm{BR}$ given in Fig. 2 assume a negligible Higgs width and currently indicate a positive deviation from expectation at Higgs masses around $120 \mathrm{GeV}$ (150 $\mathrm{GeV})$ for $\mathrm{D} \emptyset(\mathrm{CDF})$, which correspond to 2.5 (2.8) standard deviations. After considering trial factors, $\mathrm{D} \emptyset(\mathrm{CDF})$ estimate a significance of 2.0 (1.9) standard deviations to observe such an excess at any mass. Moreover, as the limits are model-independent, they are applicable to any narrow scalar decaying to $b \bar{b}$ final states produced in association with a $b$-jet. The results are translated into a MSSM exclusion in the benchmark $m_{h}^{\max }$ scenario with $\mu<0$ and include effects of the Higgs width obtained from FEYNHIGGS [8]. Such exclusions are also given in Fig. 2 (top-right, bottomright) for each experiment. The growing width for increasing $\tan \beta$ tends to spread the events over a larger region of the dijet mass spectrum and thereby yields weaker limits across the full $M_{A}$ mass range.
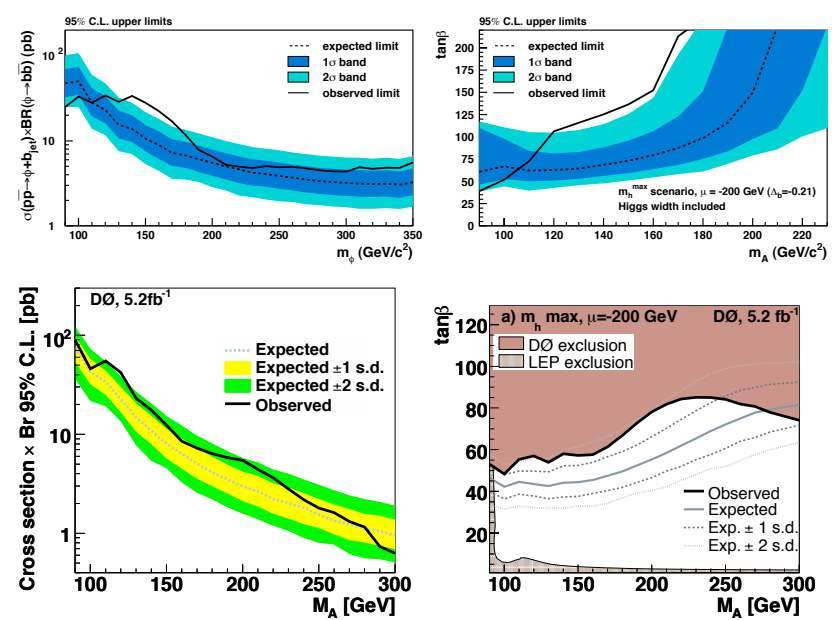

Fig. 2. Search for $\phi b \rightarrow b \bar{b} b$. 95\% C.L. results for the modelindependent limits on the production cross section multiplied by the branching ratio to $b \bar{b}$-pairs by CDF (top-left) and by DØ (bottom-left). Corresponding $\tan \beta$ exclusions in the $m_{h}^{\max }$ and $\mu<0$ scenario are given for CDF (top-right) and DØ (bottom-right).

\section{$2.3 \phi b \rightarrow \tau \tau b$ Searches}

A separate search in the $\phi b \rightarrow \tau \tau b$ channel has been performed by $\mathrm{D} \varnothing$ considering 3.7 and $7.3 \mathrm{fb}^{-1}$ of data in the $\tau_{e} \tau_{\text {had }} b$ [9] and $\tau_{\mu} \tau_{\text {had }} b$ [10] channels, respectively. Since the final state contains a $\tau \tau$-pair with an additional $b$-quark, the techniques developed for both the $\phi \rightarrow \tau \tau$ and $\phi b \rightarrow b \bar{b} b$ searches are used. The backgrounds are initially dominated by $Z \rightarrow \tau \tau$ events as well as those from multijet and $t \bar{t}$ processes. In order to discriminate between each, multivariate techniques are applied. In particular, the NN-based $b$ tagging algorithm helps suppress $Z+$ jets events while two additional signal-to-background discriminants respectively separate $t \bar{t}$ and multijet events. In the absence of any excess in data, regions of $\tan \beta \sim 45$ (25) at $M_{A}<160 \mathrm{GeV}$ are excluded in the MSSM parameter space for the $\tau_{e} \tau_{\text {had }} b$ $\left(\tau_{\mu} \tau_{\text {had }} b\right)$ channels. In fact, as shown in Fig. 3 (top), results from the $\tau_{\mu} \tau_{\text {had }} b$ mode represent the most stringent limits to date in a direct search at the Tevatron.

\subsection{Combined $\phi b \rightarrow \tau \tau b$ and $\phi b \rightarrow b \bar{b} b$ Limits}

Recently, DØ performed a MSSM Higgs combination using inputs from its $\phi b \rightarrow \tau_{\mu} \tau_{\text {had }} b$ and $\phi b \rightarrow b \bar{b} b$ searches discussed in Sects. 2.2 and 2.3 [11]. The cross section limits, as shown in Fig. 3 (bottom), are calculated assuming a narrow Higgs with respect to the experimental resolution and the sum rule $\operatorname{BR}(\phi \rightarrow \tau \tau)+\operatorname{BR}(\phi \rightarrow b \bar{b})=1$. The study is completed for $\operatorname{BR}(\phi \rightarrow \tau \tau)=0.06,0.10$, and 0.14 , and yields limits which tend to be dominated by the tau decay mode for masses up to $M_{A} \simeq 180 \mathrm{GeV}$ while at higher masses, the $3 b$ mode contributes from the decreasing dependence on the tau branching ratio.

\section{Doubly-Charged Higgs Bosons}

DØ recently performed a new search for doubly-charged Higgs bosons $\left(H^{ \pm \pm}\right)$in hadronic tau final states using 7.0 

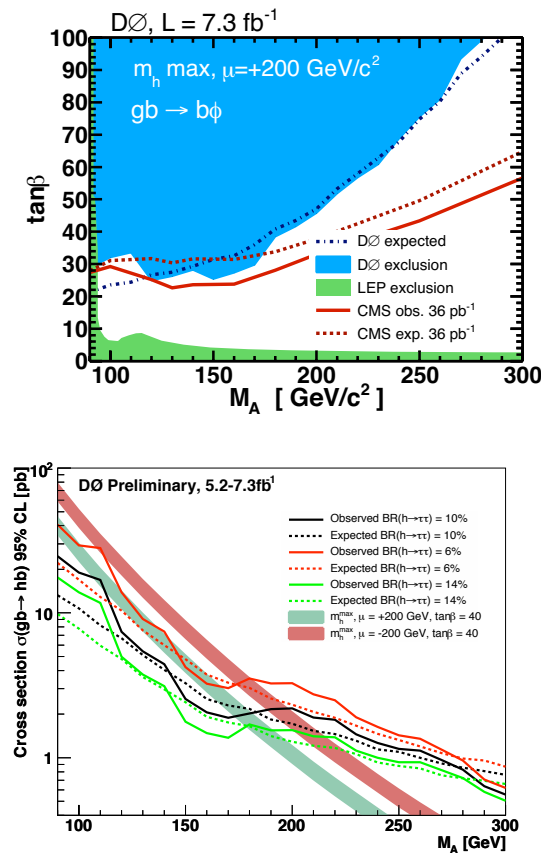

Fig. 3. DØ results at the $95 \%$ C.L. for $\left(M_{A}, \tan \beta\right)$ exclusion in the MSSM $m_{h}^{\max }$ and $\mu>0$ scenario in the $\phi b \rightarrow \tau_{\mu} \tau_{\text {had }} b$ channel (top), and the cross section limits with respect to various tau branching ratios for its combination with the $\phi b \rightarrow b \bar{b} b$ channel (bottom).

$\mathrm{fb}^{-1}$ of data [12]. Here, $H^{ \pm \pm}$are predicted in an extended Higgs sector, and in particular, the decays $H^{ \pm \pm} \rightarrow \tau^{ \pm} \tau^{ \pm}$ dominate in $S U(3)_{c} \times S U(3)_{L} \times U(1)_{Y}$ models. Moreover, an approximately equal BR for $H^{ \pm \pm} \rightarrow \tau \tau, \mu \mu, \mu \tau$ final states arises from the normal hierarchy of neutrino masses within the Higgs triplet model based on a seesaw neutrino mass production mechanism.

The search considers pair-production of $H^{ \pm \pm}$and selects events with at least one $\mu$ and two $\tau_{\text {had }}$ candidates. In order to increase the sensitivity to signal, events are categorized according to the fractional difference in background composition depending on the charge between the $\tau \tau$-pairs $\left(q_{\tau_{1,2}}\right)$. Samples with $q_{\tau_{1}}=q_{\tau_{2}}$ are dominated with $Z \rightarrow \tau \tau+$ jets background events, where a jet mimics a $\tau_{\text {had }}$ from the $Z$ decay, whereas those with $q_{\tau_{1}}=-q_{\tau_{2}}$ are mainly populated with $W Z \rightarrow \mu v e^{+} e^{-}$, where electrons are misidentified as taus. The distributions of the invariant mass of the two leading $\tau$ candidates for each sample are given in Fig. 4. Since the data agrees with the background expectation, lower limits on $M_{H^{ \pm \pm}}$are determined at the $95 \%$ C.L. for different branching ratios of the $H^{ \pm \pm}$ decays. Indeed for $\mathrm{BR}\left(H^{ \pm \pm} \rightarrow \tau^{ \pm} \tau^{ \pm}\right)=1.0$, observed (expected) limits of $M_{H^{ \pm \pm}}>128$ (116) $\mathrm{GeV}$ are set and provide the most stringent limits to date in this final state.

\section{Searches in Hidden Valley Models}

Considering $3.2 \mathrm{fb}^{-1}$ of data, CDF searched for heavy metastable particles that decay into quarks and hadronize into jets with large displaced secondary vertices [13]. The analysis uses the phenomenological Hidden Valley (HV) model as a benchmark and studies Higgs bosons decaying to two HV particles each which subsequently decay into two $b \bar{b}$ quark pairs. In order to increase acceptance, events with
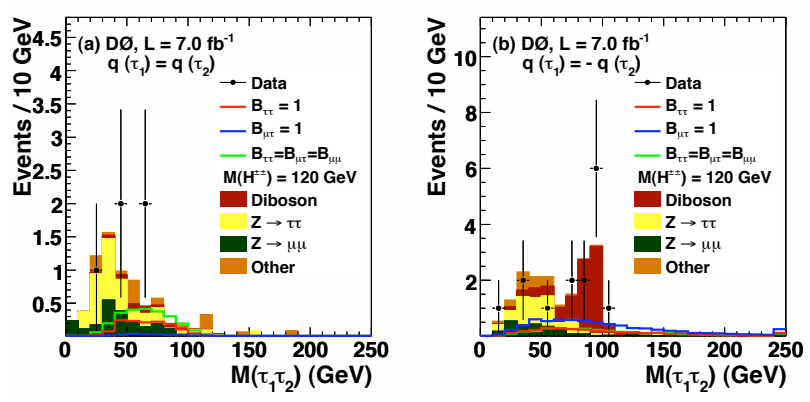

Fig. 4. Invariant mass distributions of the two leading $\tau$ candidates, $M\left(\tau_{1}, \tau_{2}\right)$, for the like (left) and opposite (right) charged samples in the doubly-charged Higgs boson search by DØ.

three or more jets are selected using a modified secondary vertex ( $b$-tagging) algorithm to account for the large HV decay length $[O(\sim 1 \mathrm{~cm})]$. Such reconstructed displaced vertices require developing two new variables in order to optimize the discrimination between the signal and background processes: the jet impact parameter, $\psi$, and the decay vertex of the HV particle, $\zeta$. Signal events tend to have more positive values of $\psi$ and $\zeta$ than negative, while background events have $\psi$ and $\zeta$ uniformly distributed around zero.

The data are split into a low (high) HV mass, $M_{H V}=$ 20 (40) GeV, search and in each, no statistically significant excess is observed in data. Therefore, 95\% C.L. $\sigma \times \mathrm{BR}$ limits are calculated in the $\mathrm{HV}$ model for different Higgs masses between $130 \leq M_{H} \leq 170 \mathrm{GeV}$ and $\mathrm{HV}$ particle lifetimes between $0.3 \leq c \tau_{H V} \leq 5 \mathrm{~cm}$. Results for the latter are shown in Fig. 5 for $M_{H}=130 \mathrm{GeV}$ and $M_{H V}=40 \mathrm{GeV}$.

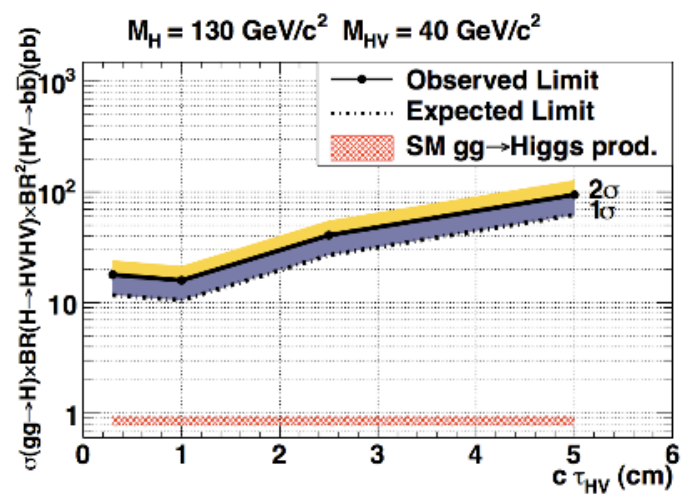

Fig. 5. Lifetime-dependent limits at the $95 \%$ C.L. on $\sigma \times$ BR by $\mathrm{CDF}$ for the production of Hidden Valley particles for a Higgs mass of $130 \mathrm{GeV}$ and a Hidden Valley particle mass of $40 \mathrm{GeV}$.

\section{Fermiophobic Higgs Boson}

In Fermiophobic Higgs models (FHM), the Higgs $\left(H_{f}\right)$ primarily couples to bosons such that the Higgs-to-fermion branching ratios are substantially suppressed. Moreover, production of $H_{f}$ at hadron colliders via the process $g g \rightarrow$ $H_{f}$ is suppressed, and the associated production, $W H_{f}$ and $\mathrm{ZH}_{f}$, as well as the Vector Boson Fusion (VBF) processes provide the primary production modes. For a relatively light 


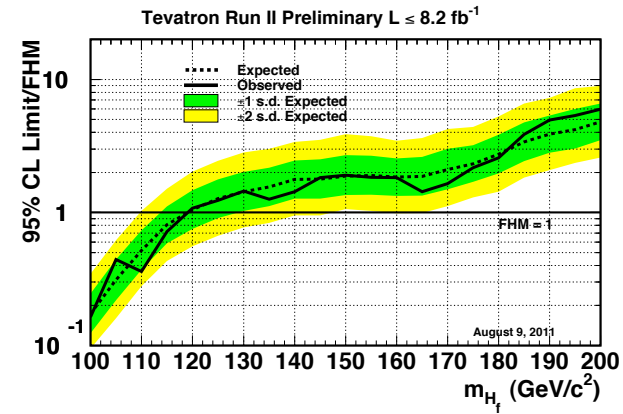

Fig. 6. Tevatron combined upper limits at the 95\% C.L. from the $\mathrm{CDF}$ and $\mathrm{D} \emptyset$ searches for Fermiophobic Higgs production.

Higgs boson, $m_{H_{f}} \lesssim 120 \mathrm{GeV}$, the Higgs predominantly decays to a $\gamma \gamma$ pair, while at higher masses, to a $W^{+} W^{-}$pair.

Both $\mathrm{D} \varnothing$ and CDF have updated earlier searches for $H_{f} \rightarrow \gamma \gamma$ by considering $8.2 \mathrm{fb}^{-1}$ [14] and $7.0 \mathrm{fb}^{-1}$ [15] of data, respectively. The CDF search applies a NN algorithm to identify photons in the central region, which increases the photon signal efficiency by $\sim 5 \%$ and background rejection by $\sim 12 \%$ compared to implementing standard cutbased photon-identification techniques. Similarly, D $\varnothing$ distinguishes photon candidates from jet backgrounds through a NN, which implements calorimeter shower-shape and preshower cluster variables. The latter is based on energyweighted cluster depositions in the preshower whose width is narrower for photons than for jets. In order to improve sensitivity, the CDF analysis separates the search into $3 p_{T}$ regions of the diphoton system: $p_{T}^{\gamma \gamma}<35,35-75$, and $>75$ $\mathrm{GeV}$. On the other hand, DØ improves sensitivity by constructing Boosted Decision Trees (BDTs) to separate the Fermiophobic signal from background. As no excess of events is observed in data for either experiment, 95\% C.L. mass-dependent limits on $\mathrm{BR}\left(H_{f}\right)$ are set. For Fermiophobic couplings, D $\varnothing$ sets a lower limit of $m_{H_{f}}>112.9 \mathrm{GeV}$ while CDF excludes masses up to $114 \mathrm{GeV}$.

The above searches in $H_{f} \rightarrow \gamma \gamma$ are subsequently combined with other CDF and D $\varnothing$ searches in the $H \rightarrow W^{+} W^{-}$ decay channels, as listed in Table 1 [16]. These searches are reinterpreted in FHM, and as shown in Fig. 6, yield a Tevatron exclusion of $m_{H_{f}}<119 \mathrm{GeV}$ at the $95 \%$ C.L., which is the most stringent limit to date on the model.

\section{Conclusions}

CDF and D $\varnothing$ have actively searched for the Higgs boson in models beyond the SM. Studies with up to $8.2 \mathrm{fb}^{-1}$ of data have been reported here. For the MSSM Higgs searches, the Tevatron has reached sensitivities of $\tan \beta \simeq 20-30$ for $M_{A}<160 \mathrm{GeV}$. Upcoming searches with larger datasets should provide further insights into the deviations from expectations which are observed in the $3 b$ decay channel by both experiments. In models with an extended Higgs sector, $\mathrm{D} \varnothing$ has performed the first search at a hadron collider for doubly-charged Higgs in $\tau \tau$ decays. Moreover, CDF's search in the Hidden Valley model can subsequently be extended to other frameworks considering differences in the production and decay kinematics. The combined Tevatron
Table 1. Fermiophobic Higgs boson search modes, corresponding mass range, and integrated luminosity contributing to the combined result by the CDF and D $\varnothing$ Collaborations given in [16].

\begin{tabular}{lll}
\hline Channel & $\begin{array}{l}\text { Mass } \\
\text { Range }[\mathrm{GeV}]\end{array}$ & $\begin{array}{l}\text { Integrated } \\
\text { Lum. }\left[\mathrm{fb}^{-1}\right]\end{array}$ \\
\hline CDF: $H_{f} \rightarrow \gamma \gamma$ & $100-150$ & 7.0 \\
CDF: $H \rightarrow W^{+} W^{-}$ & $110-200$ & 8.2 \\
CDF: $W H \rightarrow W W^{+} W^{-}$ & $110-200$ & 8.2 \\
CDF: $Z H \rightarrow Z W^{+} W^{-}$ & $110-200$ & 8.2 \\
DØ: $H_{f} \rightarrow \gamma \gamma$ & $100-150$ & 8.2 \\
DØ: $V(=W, Z) H \rightarrow \ell^{ \pm} \ell^{ \pm}+X$ & $115-200$ & 5.3 \\
\hline
\end{tabular}

search results on Fermiophobic Higgs production currently represent the most restrictive limits on its mass. The experiments look forward to the exciting results ahead with the complete dataset delivered in Run II.

\section{Acknowledgments}

The author appreciates and wishes to thank the CDF and DØ Collaborations for useful discussions and providing the results presented here.

\section{References}

1. S. Schael et al. (ALEPH, DELPHI, L3 and OPAL Collaborations), Eur. Phys. J. C. 47547 (2006).

2. T. Aaltonen et al. (CDF Collaboration), Phys. Rev. Lett. 103, 201801 (2009).

3. V. M. Abazov et al. (D0 Collaboration), Phys. Lett. B 707, 323 (2012).

4. G. Aad et al. (ATLAS Collaboration), Phys. Lett. B 705, 174 (2011).

5. S. Chatrchyan et al. (CMS Collaboration), Phys. Rev. Lett. 106, 231801 (2011).

6. T. Aaltonen et al. (CDF Collaboration), arXiv: 1106.4782 [hep-ex], submitted to Phys. Rev. D (2011).

7. V. M. Abazov et al. (D0 Collaboration), Phys. Lett. B 698, 97 (2011).

8. T. Hahn, S. Heinemeyer, W. Hollik, H. Rzehak, and G. Weiglein, Comput. Phys. Commun. 1801426 (2009); S. Heinemeyer, W. Hollik, and G. Weiglein, Comput. Phys. Commun. 124, 76 (2000). FEYNHIGGs program URL: http://www.fenyhiggs.de

9. D0 Collaboration, DØ Note 5974-CONF (2011).

10. V. M. Abazov et al. (D0 Collaboration), Phys. Rev. Lett. 107, 121801 (2011).

11. D0 Collaboration, DØ Note 6227-CONF (2011).

12. V. M. Abazov et al. (D0 Collaboration), Phys. Rev. Lett. 108, 021801 (2012).

13. T. Aaltonen et al. (CDF Collaboration), arXiv: 1109.3136 [hep-ex], submitted to Phys. Rev. D (2011).

14. V. M. Abazov et al. (D0 Collaboration), Phys. Rev. Lett. 107, 151801 (2011).

15. T. Aaltonen et al. (CDF Collaboration), arXiv: 1109.4427 [hep-ex], submitted to Phys. Rev. Lett. (2011).

16. D0 and CDF Collaborations, and Tevatron New Physics Higgs Working Group, arXiv:1109.0576 [hepex] (2011). 